\title{
An exact form of Lilley's equation with a velocity quadrupole/temperature dipole source term
}

\author{
M.E. Goldstein \\ National Aeronautics and Space Adminicration \\ Glenn Research Center \\ Cleveland, Ohio 44135
}

There have been several attempts to introduce approximations into the exact form of Lilley's equation in order to express the source term as the sum of a quadrupole whose strength is quadratic in the fluctuating velocities and a fipole whose strength is proportional to the temperature fluctuations. The purpose of this note is to show thet it is possible to choose the dependent (i.e., the pressure) variable so that this type of result can be derived directly from the Euler equations without introducing ay additional approximations.

\section{Introduction}

The subject of aeroacoustics was first put on a rational basis by Lighthill (1952, 1954) when he rearralnged the Navier-Stokes (Euler) equations into the form of a linear wave equation for a medium at rest with a quadrupole type source term. Tre crucial step in Lighthill's so called acoustic analogy approach amounts to assuming that the source term is in some sense known or that it can at least be modeled in some approximate fashion. While this approach was remarkably successful in predicting the gross features of the sound radiation from turbulent air jets, the commercial aircret industry ultimately found that they needed a much more sensitive tool that wros capable of predicting how even relatively small change in the flow would effect the $b$ radiated sound. This motivated generations of researchers to seek improvements in the Lighthill approach. Early efforts were focused on accounting for mean fiow interaction effects and there were a number of attempts to accomplish this by appiying ad hoc corrections to the original Lighthill predictions. A more satisfying approan was the one adopted by Phillips (1960), Lilley (1974) and ohers, which amounted o deriving inhomogenous moving media wave equations for the sound gerersion process.

The dominant part of the Lighthill source term is quadratic in the total flow velocity, which can be decomposed into a mean plus a fluctuating component. The source function therefore contains terms that are both linear and quadratic in the fluctuating velocity components. Lilley (1974) argued that the linear terms, which are typically such larger than the quadratic quantities, do not actually radiate any sound and should, therefore, not be included in the source function, since they would tead 0 dominate over the much smaller quadratic terms which are the true sources of soint Including them would cause the sound source to be contaminated by the small but inevitable errors resulting from the actual computation of these terms and, woaid thereby lead to inaccurate predictions of the sound field.

Unfortunately, the equation derived by Lilley has a complicated source (Colonius, Lele, and Moin, 1997) which is not of the physically expected fröm ie, the $J$ sum of a quadrupole whose strength is quadratic in the flucuating velocities and a dipole whose strength is proportional to the temperature fluctuations. There bave been a number of attempts to obtain such a source term by introducing various

This report is a preprint of an article submitted to a journal for publication. Because of changes that may be made before formal publication, this preprint is made available with the understanding that it will not be cited or reproduced without the permission of the author. 
approximations into Lilley's equation. The purpose of this note is to show thet it is possible to choose the dependent (i.e., the pressure) variable so that this type of result can be derived exactly without introducing any approximations.

\section{The Lilley Equation and Related Background Information}

Lilley (1974) showed that for an ideal gas the Navier-Stokes equations

$$
\begin{gathered}
\frac{\partial \rho}{\partial t}+\frac{\partial}{\partial x_{j}} \rho v_{j}=0 \\
\frac{\partial}{\partial t} \rho v_{i}+\frac{\partial}{\partial x_{j}} \rho v_{i} v_{j}+\frac{\partial p}{\partial x_{i}}=\frac{\partial}{\partial x_{j}} e_{i j} \\
\rho T \frac{D s}{D t}=e_{i j} \frac{\partial v_{i}}{\partial x_{j}}-\frac{\partial q_{i}}{\partial x_{i}}
\end{gathered}
$$

where

$$
s=c_{p} \ln \left(p^{1 / x} / p\right)
$$

denotes the entropy, $c_{p}$ denotes the specific heat at constant pressure $x \equiv c_{r} / c_{r}$ denotes the specific heat ratio, $t$ denotes the time, $x \equiv\left\{x_{1}, x_{2}, x_{3}\right\}$ are Cartesian constans, $p$ denotes the pressure, $\rho$ the density, $v=\left\{v_{1}, v_{2}, v_{3}\right\}$ the fluid velocity, $e_{i j}$, the viscous stress tensor, $q_{i}$ the heat flux vector and

$$
\frac{D}{D t} \equiv \frac{\partial}{\partial t}+\mathbf{v}_{j} \frac{\partial}{\partial x_{j}}
$$

is the connective derivative, can be rearranged into the third order wave equation (see, for example, Goldstein 1976, p. 253)

$$
\frac{D}{D t}\left(\frac{D^{2} \Pi}{D t^{2}}-\frac{\partial}{\partial x_{i}} c^{2} \frac{\partial \Pi}{\partial x_{i}}\right)+2 \frac{\partial v_{j}}{\partial x_{i}} \frac{\partial}{\partial x_{j}} c^{2} \frac{\partial \Pi}{\partial x_{i}}=-\frac{\partial v_{j}}{\partial x_{i}} \frac{\partial v_{k}}{\partial x_{j}} \frac{\partial v_{i}}{\partial y_{k}}+\Psi
$$

where

$$
\begin{gathered}
\Pi \equiv \frac{1}{\kappa} \ln \frac{p}{p_{o}} \\
c^{2}=\kappa R T=\kappa p / p
\end{gathered}
$$

is the squared sound speed, $R$ is the gas constant, $T$ is the temperature. $\Psi$ represents the effects of entropy fluctuations and fluid viscosity, which are generally considered to be unimportant and are therefore neglected in the following discussion.

Linearizing the velocity and thermodynamic variables about the unidirectional transversely sheared mean flow 


$$
v_{i}=\delta_{i 1} U\left(x_{2}, x_{3}\right), p=p_{o}=\text { constant } T=T_{o}\left(x_{2}, x_{3}\right)
$$

and moving the nonlinear terms to the right hand side leads to the inhomogrosous Pridmore-Brown (1957) equation

$$
\bar{L}_{o} \Pi=\Gamma
$$

where

$$
\bar{L}_{o}=\frac{D o}{D t}\left(\frac{D o^{2}}{D t^{2}}-\frac{\partial}{\partial x_{i}} c^{2} \frac{\partial}{\partial x_{i}}\right)+2 \frac{\partial U}{\partial x_{i}} \frac{\partial}{\partial x_{1}} \overline{c^{2}} \frac{\partial}{\partial x_{i}}
$$

is the Pridmore-Brown operator,

$$
\frac{D o}{D t} \equiv \frac{\partial}{\partial t}+U \frac{\partial}{\partial x_{1}}
$$

is the convective derivative based on the mean flow velocity and

$$
\overline{c^{2}}=\mathrm{k} R T_{o}
$$

Notice that

$$
\Pi=\frac{1}{\kappa} \ln \left(1+\frac{p^{\prime}}{p_{o}}\right) \simeq \frac{1}{\kappa} \frac{p^{\prime}}{p_{o}},
$$

when $\mathrm{p}^{\prime} \equiv \mathrm{p}-\mathrm{p}_{\mathrm{o}} \ll<\mathrm{p}_{\text {, }}$ i.e., when the pressure fluctuation are small $^{\mathrm{s}}$

The detailed expression for $\Gamma$ is given in Colonius, et al, (1997). This resuit is still exact but the source term is now very complicated and even more importanty does not exhibit the quadrupole form originally proposed by Lighthill (1952). Lighthill emphasized the importance of properly exhibiting the carrect multipole order of the source term before introducing specific modeling assumptions for this quantity and Colonius et al. (1997) showed the extreme sensitivity of the predicted sound field to the detailed assumptions about the form of the source.

Goldstein (1984) carried out a systematic second order asymptotic expansion and introduced a new dependent variable to show that

$$
\bar{L}_{o} \pi^{\prime}=\frac{D o}{D t} \frac{\partial f_{i}^{\prime}}{\partial x_{i}}-2 \frac{\partial U}{\partial x_{j}} \frac{\partial f_{j}^{\prime}}{\partial x_{1}}
$$

to within second order accuracy. $\bar{L}_{o}$ is defined in equation (10), the new depentent variable $\pi^{\prime}$ is defined by

$$
\pi^{\prime} \equiv \Pi+\frac{1}{2} \Pi^{2}
$$




$$
\begin{gathered}
f_{i}=-\frac{\partial}{\partial x_{j}} v_{i}^{\prime} v_{j}^{\prime}-\left(c^{2}\right) \frac{\partial \Pi}{\partial x_{i}}, \\
v_{i}^{\prime} \equiv v_{i}-\delta_{i 1} U\left(x_{2}, x_{3}\right)
\end{gathered}
$$

and

$$
\left(c^{2}\right) \equiv \kappa R\left(T-T_{o}\right)
$$

is the fluctuating sound speed.

The source term in this equation is identical to the one that would be produced by an externally applied force $f^{\prime}=\left\{f_{i}^{\prime}, f_{2}, f_{3}^{\prime}\right\}$ and is therefore property interpreted as a dipole. The first term in $f$ represents the source that would be produced by the fluctuating shear stress $v_{i}^{\prime} v_{j}^{\prime}$ and can therefore be interpreted as a quadrupole. The remaining term is a dipole source produced by the temperature fluctuations

$$
T^{\prime} \equiv T-T_{0}
$$

The quadrupole source scales like $v^{\prime} / \ell$, where $\ell$ is a characteristics length of the turbulence and the second term scales like $T^{\prime} / T_{0}\left(v^{\prime 2} / t^{\prime}\right)$, where $T^{\prime} / T_{0}$ is of the order of the turbulence Mach number squared for cold air jets, and should therefore be negligible compared to the first when the flow is subsonic (Morfey, Szewczyk, and Tester, 1978)

Colonius et al., (1997) showed that they could accurately reproduces the numerically predicted sound field radiated from a low Mach number shear layer by substituting the numerically computed values for

$$
f_{i}^{\prime}=-\frac{\partial}{\partial x_{j}} v_{i}^{\prime} v_{j}^{\prime}
$$

and $U$ into (14) and numerically solving the resulting linear equation for $\pi$. However, the Goldstein expansion, on which this result is based, is, at best, only locally valid, since nonlinear effects eventually dominate the near field disturbances and cause the expansion to breakdown. And since the acoustic field depends on the global solution to the problem, this approach does not lead to a rigorous derivation of the basic acoustic analogy equation.

\section{The Exact Equation}

The purpose of this note is to show that it is possible to obtain an exact rearrangement of the Navier-Stokes (Euler) equations that leads to a third order convective wave equation with a simple source term that still consists of a velocity quadrupole plus a fluctuating temperature dipole by introducing an appropriate dependent variable to represent the pressure fluctuations.

To this end, we neglect viscous and heat conduction effects (The final result can easily be modified to include these effects by adding an addition term to the source function.) and substitute (4) into (3) to obtain 


$$
\frac{D}{D t}\left(p^{1 / x} / p\right)=0
$$

Then multiplying equations (1) and (2) by $p^{t / x} / p$, differentiating by parts and using ( 7$)$ and (21) shows that

$$
\begin{gathered}
\frac{\partial}{\partial t} p^{1 / x}+\left(p^{1 / x_{v_{j}}}\right)=0 \\
\frac{\partial}{\partial t} p^{1 / \times} v_{i}+\frac{\partial}{\partial x_{j}}\left(p^{1 / x} v_{i} v_{j}\right)+c^{2} \frac{\partial p^{1 / x}}{\partial x_{i}}=0
\end{gathered}
$$

which upon introducing (11) and (17) can be written as

$$
\frac{D o}{D t} p^{1 / \kappa}+\frac{\partial}{\partial x_{j}}\left(p^{1 / \kappa} v_{j}^{\prime}\right)=0
$$

and

$$
\frac{D o}{D t}\left(p^{1 / \kappa} v_{i}^{\prime}\right)+\delta_{l i} p^{1 / \kappa} v_{j}^{\prime} \frac{\partial U}{\partial x_{j}}+c^{2} \frac{\partial p^{1 / k}}{\partial x_{i}}=-\frac{\partial}{\partial x_{j}}\left(p^{1 / \kappa} v_{i}^{\prime} v_{j}^{\prime}\right)
$$

where we have used equation (24) to simplify equation (25). Upon introducing the new dependent variables

$$
\begin{aligned}
& u_{i} \equiv\left(p / p_{o}\right)^{1 / x} v_{i}^{\prime} \\
& \pi \equiv\left(p / p_{o}\right)^{1 / x}-1
\end{aligned}
$$

and using (12) and (18), these become the inhomogeneous linearized Euler equarions

$$
\begin{gathered}
\frac{D o \pi}{D t}+\frac{\partial u_{j}}{\partial x_{j}}=0 \\
\frac{D o u_{i}}{D t}+\delta_{1 i} u \cdot \nabla U+\overline{c^{2}} \frac{\partial \pi}{\partial x_{i}}=f_{i},
\end{gathered}
$$

where the externally applied force $f_{i}$ is now given by

$$
f_{i}=-\frac{\partial}{\partial x_{j}}(1+\pi) v_{i}^{\prime} v_{j}^{\prime}-c^{2^{\prime}} \frac{\partial \pi}{\partial x_{i}}
$$

These are identical in form to the linearized equations discussed in Chapter 1 of Goldstein (1976), where it is shown (by taking the convective derivative of the first equations and the divergence of the second, subtracting the results and then wsing the 
second equations with $i=1$ to eliminate the velocity fluctuation on the left hand side) that they can be rearranged into the inhomogeneous Pridmore-Bown equation

$$
\bar{L}_{o} \pi=\frac{D o}{D t} \frac{\partial f_{i}}{\partial x_{i}}-2 \frac{\partial U}{\partial x_{j}} \frac{\partial f_{j}}{\partial x_{1}}
$$

which is identical to (14) but with the pressure fluctuation $x$ now given by (27) and the externally applied force $f$ now given by (30) rather than by (10) Notice that

$$
\pi \rightarrow \frac{1}{\kappa} p^{\prime} l_{p_{0}} \text { when } p^{\prime} \equiv p-p_{0} \rightarrow 0,
$$

Finally, it is worth noting that (31) can be written as

$$
L_{o} \pi=\frac{D o}{D t} \frac{\partial \tilde{f}_{i}}{\partial x_{i}}-2 \frac{\partial U}{\partial x_{i}} \frac{\partial \tilde{f}_{i}}{\partial x_{1}}
$$

where $\mathrm{L}_{0}$ is the same as (10) but with $\overline{c^{2}}$ replaced by $c^{2}=\overline{c^{2}}+c^{2}$ and

$$
\tilde{f}_{i} \equiv-\frac{\partial}{\partial x_{j}}(1+\pi) v_{i}^{\prime} v_{j}^{\prime}
$$

is now a pure quadrupole source, which suggests that the dipole (31) could also be interpreted as a weak nonlinear propagation

Aside from the definition of the pressure fluctuation, the only difference between equations (14) and (31) is the appearance of the pressure fluctuation factor $(1+\pi)$ in the quadrupole strength $(1+\pi) v_{i}^{\prime} v_{j}^{\prime}$. Since $\pi$ should be of the order of the turbulent intensity squared (which is typically small comparod to unity) and since substituting exact values of $U$ and $f_{i}$ into (31) should yield exactly the same result as the direct numerical solution for the sound field, this explains why Coloneus et al. (1997) were able to obtain such good agreement using the two different approaches.

Lighthill indicated that the basis of his acoustic analoge is the demonstration that there is an exact analogy between the density fluctuations in any real flow and those produced by a quadrupole source in an ideal stationary zcoustic medium. The present result shows that, aside from viscous and heat conduction effects, there is an exact analogy between the ( $p / p)^{1 / x}$ fluctuations in any real flow and the corresponding linear fluctuations in this quantity produced by a quadrupale phos a temperature dipole source in an arbitrary ideal transversely sheared mean flow. 


\section{References}

Colonius, T., Lele, S.K, and Moin, P., 1997, "Sound Generation in a Mixing Layer," J. Fluid mech., Vol. 330, pp. 375-409.

Goldstein, M.E., 1976 Aeroacoustics, McGraw-Hill, New York.

Goldstein, M.E., 1984, “Aeroacoustics of Turbulent Shear Flows," Annual Review of Fluid mechanics, Vol. 16, pp. 263-295.

Lighthill, M.J., 1952, "On Sound Generated Aerodynamically: I. General Theory," Proc. Roy. Soc. Lond. A, vol. 211, pp. 564-587.

Lighthill, M.J., 1954 "On Sound Generated Aerodynamically. II. Turbulence as a Source of Sound," Proc. Roy. Soc. Lond. A, Vol. 222, pp. 1-32.

Lilley, G.M., 1974, "On the Noise From Jets," Noise Mechanisms, AGARD-CP-131, pp. 13.1-13.12.

Morfey, C.L., Szewczyk, V.M., and Tester, B.J., 1978, "New Scaling Laws for Hot and Cold Jet Mixing Noise Based on a Geometrics Acoustics Model," J. Sound Vib., Vol. 61, No. 2, pp. 255-292.

Phillips, O.M., 1960, "On the Generation of Sound by Supersonic Turbulent Shear Layers," J. Fluid Mech., 9, pt. 1, 1-28.

Pridmore-Brown, D.C., 1958, "Sound Propagation in a Fluid Flowing Through an Attenuating Duct," J. Fluid Mech., Vol. 4, pp. 393-406. 